\title{
Spray dried proliposomes of Rosmarinus officinalis polyphenols: a quality by design approach
}

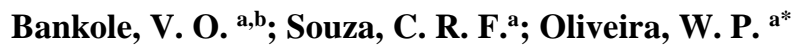

a LAPROFAR group, Faculty of Pharmaceutical Sciences of Ribeirão Preto, University of São Paulo, Ribeirão Preto, Brasil.

b Department of Pharmaceutics, Faculty of Pharmacy, Obafemi Awolowo University, IleIfe, Nigeria.

*E-mail of the corresponding author: wpoliv@fcfrp.usp.br

\begin{abstract}
Harnessing the benefits of rosemary polyphenols is limited by variability in their physicochemical properties. These limitations may be overcome by encapsulation in systems possessing hydro-lipophilic centers thereby accommodating molecules of different polarities. Proliposomes offer a viable option in this regards, being particles which form liposomal suspension in water. Lyophilized extracts of rosemary was encapsulated in hydrogenatedsoyphosphatidylcholine/cholesterol by solvent replacement method. Spray dried proliposomes were obtained with lactose as carrier. Using Central Composite Design, the effects of lipid, extract and carrier concentrations on response variables including bioactive content and retention, moisture content characteristics, recuperation and redispersibility were evaluated by statistical analysis.
\end{abstract}

Keywords: Polyphenols; antioxidant; proliposomes; spray drying. 


\section{Introduction}

Plant polyphenols have indeed proven to be very worthy antioxidants with evidence of protection of cell constituents against oxidative damage, therefore, limiting the risk of various degenerative diseases associated to oxidative stress. ${ }^{[1]}$ This efficacy together with their safety profile, biocompatibility, multifunctionality, relative affordability and ready availability may have favoured their choice as additives in foods and nutraceticals. ${ }^{[2]}$ Research into polyphenol has garnered much momentum over the years. ${ }^{[3]}$ One important vegetable source of these polyphenol compounds is Rosmarinus officinalis L. (Lamiaceae) commonly called rosemary. Extracts of rosemary have been evaluated for antioxidant and various other effects, and its efficacy correlated to high amounts of phenolic acids e.g. rosmarinic acid and caffeic acid; phenolic diterpenes being chiefly carnosic acid and carnosol; and flavonoids. ${ }^{[4]}$ The performance of obtained extract is influenced by processing and environmental conditions to which it is exposed as well as its physicochemical properties. ${ }^{[5]}$ Thus, employment of strategies that ensure incorporation of the multiple bioactive polyphenol components of these extracts for synergistic activity while desirable physicochemical properties are kept intact is evidently desirable. The aim of this study, therefore, is to use Design of Experiments (DoE) in evaluating the effects of composition factors critical to desirable response attributes and system performance during spray drying of proliposomes of rosemary polyphenols.

\section{Materials and Methods}

\subsection{Preparation of rosemary extract}

Polyphenol-rich powder extracts of rosemary was obtained by freeze drying. Concentrated liquid extract was congealed at -20 and $-80^{\circ} \mathrm{C}$ then lyophilized using VLP 195 FD-115, Thermo Fischer Scientific Lyophilizer at $-40{ }^{\circ} \mathrm{C}$. Dry rosemary powder particles obtained was collected in airtight amber bottles and stored at $-20^{\circ} \mathrm{C}$.

\subsection{Quantification of marker compounds in lyophilized extract by HPLC}

Polyphenol markers, namely caffeic acid (CA), rosmarinic acid (RA), carnosol (CAR), and carnosic acid (CNA) in the powdered extract were quantified by HPLC. The chromatographic method used was previously developed and validated by our group. ${ }^{[6]}$

\subsection{DoE for evaluation of effect of composition variables in proliposome formulations}

Control parameters critical to physicochemical characteristics of proliposome powders were selected using Quality by Design (QbD) approach,with a focus on composition variables. Critical independent variables evaluated were concentration of lipid, extract and drying carrier (relative to liposomal solid content). The effects of these factors on quality attributes of proliposomes such as biomarker retention, biomarker content, water activity and moisture content were investigated. The individual and combined effects of these factors were evaluated at three levels using a completely randomized $2^{3}$ Box-Wilson Central 
Bankole, V.O.; Souza, C.R.F; Oliveira, W.P.

Composite Design (CCD), ${ }^{[7]}$ with three replicates in the central point. Tables 1 and 2 show the uncoded and coded variables studied, respectively.

Table 1: Uncoded variables and their respective values

\begin{tabular}{ccccc}
\hline Coded variable & Uncoded variables & -1 & 0 & 1 \\
\hline A & Lipid concentration (\%) & 4 & 7 & 10 \\
B & Extract concentration (\%) & 1.5 & 3 & 4.5 \\
C & Carrier concentration (\%) & 1 & 1.2 & 1.4 \\
& i.e. Carrier:(Lipid+extract) & & & \\
\hline
\end{tabular}

Table 2: CCD for proliposomes of rosemary (coded variables)

\begin{tabular}{lccc}
\hline & \multicolumn{3}{c}{ Coded variables } \\
\cline { 2 - 4 } Formulation & $\mathrm{A}$ & $\mathrm{B}$ & $\mathrm{C}$ \\
\hline F2 & 1.000 & -1.000 & -1.000 \\
F9 & -1.682 & 0.000 & 0.000 \\
F4 & 1.000 & 1.000 & -1.000 \\
F3 & -1.000 & 1.000 & -1.000 \\
F11 & 0.000 & -1.682 & 0.000 \\
F13 & 0.000 & 0.000 & -1.682 \\
F10 & 1.682 & 0.000 & 0.000 \\
F16 & 0.000 & 0.000 & 0.000 \\
F1 & -1.000 & -1.000 & -1.000 \\
F12 & 0.000 & 1.682 & 0.000 \\
F15 & 0.000 & 0.000 & 0.000 \\
F7 & -1.000 & 1.000 & 1.000 \\
F17 & 0.000 & 0.000 & 0.000 \\
F5 & -1.000 & -1.000 & 1.000 \\
F6 & 1.000 & -1.000 & 1.000 \\
F8 & 1.000 & 1.000 & 1.000 \\
F14 & 0.000 & 0.000 & 1.682 \\
\hline
\end{tabular}

The process model has the following form (Eq. 1):

$$
\begin{gathered}
Y i=a_{0}+a_{1} \cdot X_{1}+a_{2} \cdot X_{2}+a_{3} \cdot X_{3}+a_{11} \cdot X_{1}^{2}+a_{22} \cdot X_{2}^{2}+a_{33} \cdot X_{3}^{2} \\
+a_{12} \cdot X_{1} \cdot X_{2}+a_{13} \cdot X_{1} \cdot X_{3}+a_{23} \cdot X_{2} \cdot X_{3}+\varepsilon
\end{gathered}
$$

where: $\mathrm{a}_{0}$ to $\mathrm{a}_{33}$ are the regression coefficients, $\mathrm{X}_{1}$ to $\mathrm{X}_{3}$ denotes the factors, $\mathrm{Y}_{\mathrm{i}}$ is the relative average or expected response associated with the combination factors and $\varepsilon$ represents the experimental error. Statistical significance of linear, quadratic and interaction effects of the investigated variables on evaluated proliposomes properties was assessed through variance analysis (ANOVA) and regression analysis using Statistica ${ }^{\circledR} 10$ (StatSoft Inc, USA). 


\subsection{Preparing proliposomes of polyphenol-rich rosemary extract}

\subsubsection{Incorporation of rosemary polyphenols}

Liposomal preparations incorporating polyphenols of rosemary were prepared by solvent replacement method. ${ }^{[8,9]}$ The lipid phase comprised predetermined quantities (by DoE) of hydrogenated soy phosphatidyl choline (Phospolipon 90H - LIPOID GMBH, Ludwigshafen, Germany) and cholesterol (Sigma-Aldrich, St Louis, USA). The aqueous phase consists of a dispersion of lyophilized rosemary extract in purified water. The two phases were brought to the same temperature before the lipid phase was injected into the aqueous phase under agitation. Residual solvent was removed by rotary evaporation at 48 ${ }^{\circ} \mathrm{C} / 600 \mathrm{mmHg}$. Liposomal formulation obtained was dried with lactose (Natural Pharma, $\mathrm{SP}$, Brasil) as the drying carrier (quantity determined by CCD).

\subsubsection{Spray drying}

Spray dried proliposomes (SDP) were generated from the liquid liposomal formulation (LLF) in a laboratory scale bench top SD-05 spray dryer (Lab-Plant UK Ltd, Huddersfield, UK) with a concurrent flow regime. Spray drying conditions were maintained as follows: atomizer, $1 \mathrm{~mm}$; inlet drying gas temperature, $100{ }^{\circ} \mathrm{C}$; feed flow rate of liquid formulation, $4 \mathrm{~g} / \mathrm{min}$; atomizing air pressure, $1.5 \mathrm{KgF} / \mathrm{cm}^{2}$; feed flow rate of atomizing air, $17 \mathrm{Lpm}$; and feed flow rate of spray drying air, $60 \mathrm{~m}^{3} / \mathrm{h}$.

\subsection{Proliposome characterization}

Proliposome powders were characterized as follows:

\subsubsection{Water activity (Aw) and moisture content (Xp)}

Moisture content of the spray dried product was determined in a moisture analyzer Sartorius MA35 (Goettingen, Germany). Water activity was measured in an AquaLab 4TEV (Decagon Devices Inc., Pullman, WA) at $25^{\circ} \mathrm{C}$, using dew point sensor.

\subsubsection{Retention and content of biomarker compounds in proliposomes}

Total retention and concentration of bioactive markers in proliposomes was evaluated by the HPLC quantification method described. ${ }^{[6]}$ Retention was determined as \% biomarker compound quantified in a sample of proliposome powder relative to amount in lyophilized extract contained in the proliposome sample. Total content of biomarker in bulk quantity was determined relative to proliposome powder (w/w). Concentrations were determined by comparison to those of standards.

\section{Results and Discussion}

\subsection{Proliposome characterization}

Experimental data of proliposome properties evaluated were subjected to regression analysis to detect the statistically significant effects of composition variables on proliposomes properties. In this way, the linear, quadratic and interaction regression 
coefficients and their statistical significance were derived (Table 3). ANOVA and effect estimates assume normal and independent residuals distribution, with mean zero and constant variance.

\subsubsection{Water activity ( $\mathrm{Aw}$ ) and Moisture content (Xp)}

Water activity $\left(A_{w}\right)$, a measure of the energy state of water present in a system, is a qualitative property independent of sample quantity. It is usually considered as indicative of microbiological stability with values less than 0.5 recommended as enough to guarantee product stability. Results showed that besides the interaction effects between lipid/extract concentration and extract/carrier concentration, only the quadratic effect of carrier concentration also had significant effect on water activity (Table 3). The negative value attributed to the effect may be that water activity is related to the form in which the lactose exists, among other factors. Lactose has been previously used in drying of lipid systems encapsulating polyphenols. ${ }^{[10]}$ Lactose monohydrate has been shown to lose its water of hydration at $100{ }^{\circ} \mathrm{C}$, the drying temperature in this study. ${ }^{[11]}$ Thus, increasing the carrier concentration leads to a higher percentage bound-water loss and lower $\mathrm{A}_{\mathrm{w}}$. While Xp and $A_{w}$ are mainly related to the drying conditions, formulation composition also plays an important role as drying could promote changes in water binding and dissociation, with effect product properties. On the other hand, carrier concentration showed no level of significance on Xp of SDP while lipid and extract concentrations became significant only at $\mathrm{p} \leq 0.1$. Values of $\mathrm{A}_{\mathrm{w}}$ and $\mathrm{Xp}$ were $<0.5$ and 5\%, respectively, for SDP (data not shown). This suggests that proliposomes obtained are potentially stable to microbial proliferation.

\subsubsection{Retention of selected biomarkers}

Retention of RA and CAR in SDP showed significant dependence on similar factors. Linear effects of $\%$ extract was observed as highly significant $(p \leq 0.01)$ for retention of both compounds. However, while the effect on RA was positive, it was negative for CAR, implying that as the quantity of extract incorporated increases, retention of the former increases while that of the latter decreases. The degradation pattern of CAR in this system appears to be concentration dependent thus giving rise to decreased \% retention on increasing concentration, ${ }^{[12]}$ typical of first order degradation. Its higher lipophilicity also suggests favored partitioning into lipidic wall of the proliposomes rather than aqueous core.

Hence, higher extract/lipid ratio favourably retained hydrophilic compounds protected in the aqueous vesicle core against lipophilic components which are no longer efficiently encapsulated and therefore exposed to degradation at the vesicle periphery. ${ }^{[13]}$ The linear effect of \% lipid incorporated further demonstrates this relationship, although quasi significant $(\mathrm{p} \leq 0.10)$ to both RA and CAR retention with respective negative and positive effects. It may be that the negative effect of \% lipid on RA retention is related to solubility of the compound. Increasing lipid concentration is suggested to favor retention of lipophilic material such as CAR (positive value for same factor) as against less lipophilic ones such as RA. Evaluated lone factors ranged from being significant $(\mathrm{p} \leq 0.05)$ to highly significant ( $\mathrm{p}$ $\leq 0.01$ ) for CNA retention. Lipid and carrier concentrations showed positive effects on 
CAN, similar to CAR and in line with their lipophilicity. Increasing \% extract, however, significantly $(\mathrm{p}<0.05)$ reduces relative retention of CAN. Since the degradation of CAR is concentration dependent, the reaction is skewed away from buildup of its concentration hence, further degradation of CNA. The significant $(\mathrm{p}<0.05)$ positive effect of carrier concentration on CNA retention may be due to protective effect offered by lactose molecules to prevent or slow down it`s degradation. ${ }^{[10,14]}$ Evaluated factors showed no interactive effect on retention properties of bioactive compounds in SDP. In any case, the integrity of the bioactive compounds was largely preserved with proliposome products exhibiting $60.0 \%-104.6 \%$ retention. It is noted that these retention extremes is in accordance with the lipophilicity of each compound i.e. more lipophilic compounds such as CAR and CNA have the highest retention values at F11 with the highest lipid ratio whereas the less hydrophobic compound, RA is more concentrated at F9 with least lipid ratio, and vice versa (Table 4). Response surface plots (not shown) for visual effects of relationship between studied variables and biomarker retention response revealed that retention pattern of marker compounds at 0.0 (mean) level of Carrier/ $\mathrm{C}_{\text {solid }}$ is similar to that at -1.682 (low), and 1.682 (high) levels. While RA retention is facilitated at high level of \% extract, both CAR and CNA are favourably retained at high levels of \% lipid.

Table 3: Linear, quadratic and interaction regression coefficients and statistical significance for SDP

\begin{tabular}{|c|c|c|c|c|c|c|c|c|}
\hline \multirow{3}{*}{ Formulation } & \multicolumn{8}{|c|}{ Regression Coefficients for Selected Responses } \\
\hline & \multicolumn{3}{|c|}{$\begin{array}{c}\text { Biomarker Retention } \\
\text { (\%) }\end{array}$} & \multicolumn{3}{|c|}{$\begin{array}{l}\text { Total Biomarker content } \\
\text { (mg/100g) }\end{array}$} & \multirow[t]{2}{*}{$\begin{array}{l}A_{w} \\
(-)\end{array}$} & \multirow[t]{2}{*}{$\begin{array}{c}\text { Xp } \\
(\%)\end{array}$} \\
\hline & RA & CAR & CNA & RA & CAR & CNA & & \\
\hline$a_{0}-$ Mean/Interc. & $97.342^{*}$ & $88.284^{*}$ & $80.157^{*}$ & $116.776^{*}$ & $88.472^{*}$ & $73.005^{*}$ & $0.402^{*}$ & $1.837^{*}$ \\
\hline$a_{1}-\mathrm{A}_{(\mathrm{L})}$ & $-3.587^{* * *}$ & $4.827^{* * *}$ & $4.741^{*}$ & $-45.242^{*}$ & $-22.403^{*}$ & $-19.385^{*}$ & -0.001 & $0.239^{* * *}$ \\
\hline$a_{11}-\mathrm{A}_{(\mathrm{Q})}$ & 1.268 & -4.116 & -1.192 & $15.738^{*}$ & 3.249 & $5.187^{*}$ & 0.009 & $0.290^{* * *}$ \\
\hline$a_{2}-\mathrm{B}_{(\mathrm{L})}$ & $7.058^{*}$ & $-12.941^{*}$ & $-3.383^{*}$ & $47.765^{*}$ & $24.194^{*}$ & $23.725^{*}$ & -0.008 & -0.193 \\
\hline$a_{22}-\mathrm{B}_{(\mathrm{Q})}$ & $-4.261^{* *}$ & $6.494^{* * *}$ & -0.715 & $-7.387^{* * *}$ & $-5.343^{* *}$ & $-5.788^{*}$ & 0.004 & $0.260^{* * *}$ \\
\hline$\left.a_{3}-\mathrm{C}_{(\mathrm{L})}\right)$ & 0.013 & -0.292 & $2.175^{* *}$ & $-11.404^{*}$ & $-8.819 *$ & $-5.249^{*}$ & 0.000 & -0.101 \\
\hline$a_{33}-\mathrm{C}_{(\mathrm{Q})}$ & 0.983 & -1.050 & $1.994^{* *}$ & 0.136 & 1.418 & $2.513^{* *}$ & $-0.023^{* *}$ & 0.161 \\
\hline$a_{12}-\mathrm{A}_{(\mathrm{L})} * \mathrm{~B}_{(\mathrm{L})}$ & 1.061 & 0.162 & 0.674 & $-8.809^{* * *}$ & -2.817 & $-1.958^{* * *}$ & $0.023^{* *}$ & $0.296^{* * *}$ \\
\hline$a_{13}-\mathrm{A}_{(\mathrm{L})} * \mathrm{C}_{(\mathrm{L})}$ & 0.710 & -1.565 & 0.965 & 4.220 & 1.963 & $1.748^{* * *}$ & -0.015 & -0.216 \\
\hline$a_{23}-\mathrm{B}_{(\mathrm{L})} * \mathrm{C}_{(\mathrm{L})}$ & -0.832 & -2.424 & -0.740 & -5.070 & $-5.643^{* * *}$ & $-1.801^{* * *}$ & $-0.025^{* *}$ & -0.192 \\
\hline $\mathrm{R}^{2} \mathrm{adj}$ & 0.744 & 0.720 & 0.853 & 0.967 & 0.951 & 0.992 & 0.824 & 0.751 \\
\hline
\end{tabular}

${ }^{*}$ Effect significant at $\alpha \leq 0.01 ;{ }^{* *}$ Effect significant at $\alpha \leq 0.05 ;{ }^{* * *}$ Effect significant at $\alpha \leq 0.1$

\subsubsection{Biomarker composition of proliposomes}

Linear effects of lipid and extract concentrations were most significant $(p \leq 0.001)$ of factors evaluated. This being that the overall content of polyphenols components of proliposome product is directly proportional to the amount of extract incorporated and inversely to the lipid concentration and is consistent with results obtained for other extracts incorporated in lipid systems. Regression coefficients presented (Table 3) showed that whereas \% extract has positive values for all compounds, those of \% lipid are negative. $\mathrm{R}^{2}$ values indicate the adequacy of adjustment of results obtained by the statistical model proposed. Carrier concentration was also highly significant $(\mathrm{p} \leq 0.01)$ to concentrating all marker compounds in the proliposome. Lone effects of all factors evaluated showed 
different levels of significance. Whereas interactions between \% lipid and \% extract, and \% extract and carrier concentration showed quasi significance $(\mathrm{p} \leq 0.1)$ on the total content of RA and CNA, the same level of significance was observed on CNA content following interaction between \% lipid and carrier concentration. These results reveal that polyphenols accumulation is highly dependent on the \% extract present and independent of the levels of concentration of carrier. Surface plots (not shown) show effects of the most significant variables (\%extract and \% lipid) remained visibly constant at all Carrier/C Solid $_{\text {levels. }}$

Table 4: Retention and total concentration of biomarkers in spray dried SDP

\begin{tabular}{|c|c|c|c|c|c|c|}
\hline \multirow[t]{2}{*}{ Formulation } & \multicolumn{3}{|c|}{$\begin{array}{c}\text { Biomarker retention in SDP } \\
(\%)\end{array}$} & \multicolumn{3}{|c|}{$\begin{array}{c}\text { Total biomarker content of SDP } \\
(\mathrm{mg} / \mathbf{1 0 0 g})\end{array}$} \\
\hline & $R A$ & CAR & CNA & $R A$ & CAR & CNA \\
\hline F1 & $97.2 \pm 2.8$ & $88.1 \pm 1.8$ & $77.4 \pm 1.7$ & $579.9 \pm 16.6$ & $443.7 \pm 9.0$ & $355.7 \pm 8.0$ \\
\hline F2 & $86.1 \pm 0.8$ & $101.3 \pm 2.4$ & $82.0 \pm 0.9$ & $245.9 \pm 2.2$ & $244.2 \pm 5.8$ & $180.2 \pm 2.0$ \\
\hline F3 & $102.9 \pm 2.2$ & $83.2 \pm 2.0$ & $71.1 \pm 0.5$ & $1191.6 \pm 25.8$ & $814.1 \pm 19.5$ & $634.4 \pm 4.2$ \\
\hline F4 & $97.8 \pm 1.0$ & $83.7 \pm 1.0$ & $77.8 \pm 0.5$ & $664.2 \pm 6.8$ & $479.9 \pm 6.0$ & $406.7 \pm 2.6$ \\
\hline F5 & $96.9 \pm 0.7$ & $103.0 \pm 1,6$ & $84.0 \pm 0.9$ & $481.9 \pm 3.4$ & $432.6 \pm 6.5$ & $321.8 \pm 3.3$ \\
\hline F6 & $90.5 \pm 1.9$ & $96.6 \pm 2.4$ & $91.9 \pm 3.5$ & $215.1 \pm 4.6$ & $193.9 \pm 4.9$ & $168.3 \pm 6.4$ \\
\hline F7 & $101.0 \pm 1.2$ & $75.1 \pm 0.9$ & $74.2 \pm 0.3$ & $975.1 \pm 11.3$ & $611.8 \pm 7.0$ & $551.5 \pm 2.0$ \\
\hline F8 & $97.1 \pm 0.7$ & $82.7 \pm 1.7$ & $85.3 \pm 1.5$ & $549.3 \pm 3.9$ & $395.1 \pm 8.3$ & $371.8 \pm 6.6$ \\
\hline F9 & $106.4 \pm 1.5$ & $62.0 \pm 0.4$ & $66.2 \pm 1.1$ & $1281.9 \pm 18.1$ & $630.8 \pm 3.9$ & $614.1 \pm 9.9$ \\
\hline F10 & $93.0 \pm 0.8$ & $92.4 \pm 1.3$ & $86.8 \pm 0.5$ & $369.0 \pm 3.1$ & $309.3 \pm 4.5$ & $265.0 \pm 1.5$ \\
\hline F11 & $63.8 \pm 2.6$ & $104.6 \pm 0.9$ & $83.6 \pm 2.2$ & $80.9 .0 \pm 3.3$ & $150.7 \pm 0.9$ & $81.7 \pm 2.2$ \\
\hline F12 & $104.4 \pm 1.8$ & $73.8 \pm 0.5$ & $72.1 \pm 0.8$ & $916.0 \pm 16.0$ & $546.3 \pm 3.9$ & $487.0 \pm 5.2$ \\
\hline F13 & $99.3 \pm 0.1$ & $87.4 \pm 0.1$ & $84.7 \pm 1.0$ & $699.6 \pm 5.1$ & $519.5 \pm 4.8$ & $459.6 \pm 5.5$ \\
\hline F14 & $98.5 \pm 2.3$ & $84.4 \pm 1.1$ & $86.3 \pm 1.8$ & $510.1 \pm 12.0$ & $368.7 \pm 4.8$ & $343.8 \pm 7.3$ \\
\hline F15 & $98.1 \pm 1.1$ & $88.5 \pm 1.4$ & $79.2 \pm 1.5$ & $585.4 \pm 6.5$ & $425.9 \pm .3$ & $364.1 \pm 7.0$ \\
\hline F16 & $97.3 \pm 1.2$ & $88.4 \pm 1.2$ & $80.4 \pm 0.6$ & $580.9 \pm 7.2$ & $465.6 \pm 6.2$ & $369.3 \pm 2.6$ \\
\hline F17 & $97.0 \pm 1.0$ & $87.7 \pm 0.7$ & $81.0 \pm 0.5$ & $578.8 \pm 6.0$ & $441.8 \pm 3.3$ & $381.3 \pm 2.5$ \\
\hline
\end{tabular}

\section{Conclusions}

Proliposome is a viable system for the retention of polyphenols of rosemary. Careful choice of carriers and their appropriate concentration may, however, be necessary towards obtaining these desirable properties. These many factors and possibilities underscore the importance of Design of Experiments, a systematic approach to determine the relationship between factors involved in a process and the effects of those factors on the output of that process. The Central Composite Design is demonstrated as an efficient approach in which the effects of several factors can be assessed within a workable number of experiments.

\section{References}

[1] Scalbert, A.; Manach, C.; Morand, C.; Rémésy, C.; Jiménez, L. Dietary polyphenols and the prevention of diseases. Critical Reviews in Food Science and Nutrition 2005, 45 (4), 287-306.

[2] Ozsoy, N.; Yilmaz-ozden, T.; Serbetci, T.; Kultur, S.; Akalin, E. Antioxidant, antiinflammatory, acetylcholinesterase and thioredoxin reductase inhibitory activities of 
nine selected Turkish medicinal plants. Indian Journal of Traditional Knowledge 2017, 16 (4), 553-561.

[3] Cortés-Rojas, D.F.; Souza, C.R.F.; Oliveira, W.P. Assessment of stability of a spray dried extract from the medicinal plant Bidens pilosa L. Journal of King Saud University - Engineering Science 2016, 28 (2), 141-146.

[4] Tavassoli, S.K.; Mousavi, S.M.; Emam-Djomeh, Z.; Razavi, S.H. Chemical composition and evaluation of antimicrobial properties of Rosmarinus officinalis L. essential oil. African Journal of Biotechnology 2011, 10 (63), 13895-13899.

[5] Taghvaei, M.; Jafari, S.M. Application and stability of natural antioxidants in edible oils in order to substitute synthetic additives. Journal of Food Science and Technology 2015, 52 (3), 1272-1282.

[6] Souza, C.R.F. Production of standardized dried extracts of Brazilian medicinal plants: study of the technical and economic feasibility of the process in spout bed $(\mathrm{PhD}$ Thesis) 2007, 1-32.

[7] NIST/SEMATECH e-Handbook of Statistical Methods, http://www.itl.nist.gov/div898/handbook/, 2017.

[8] Wagner, A.; Vorauer-Uhl, K. Liposome Technology for Industrial Purposes. Journal of Drug Delivery 2011, 1-9.

[9] Hasan, M.; Hasan, M.; Mondal, J.C.; Hasan, M.Al., Talukder, S.; Rashid, H.A. Liposomes: An advance tools for novel drug delivery system. The Pharma Innovation Journal 2017, 6 (11), 304-311.

[10] Secolin, V.A.; Souza, C.R.F.; Oliveira, W.P. Spray drying of lipid-based systems loaded with Camellia sinensis polyphenols. Journal of Liposome Research 2017; 27 (1), 11-20.

[11] Raut, D.M.; Allada, R.; Pavan, K.V. ; Deshpande, G.; Patil, D.; Patil, A.; Deshmukh, A.; Sakharkar, D. M.; Bodke, P.S.; Mahajan, D.T. Dehydration of Lactose monohydrate: Analytical and physical characterization. Der Pharmacia Lettre 2011, 3 (5), 202-212.

[12] Zhang, Y.; Smuts, J.P.; Dodbiba, E.; Rangarajan, R.; Lang, J.C.; Armstrong, D.W. Degradation study of carnosic acid, carnosol, rosmarinic acid, and rosemary extract (Rosmarinus officinalis L.) assessed using HPLC. Journal of Agricultural and Food Chemistry 2012, 60 (36), 9305-9314.

[13] Nakayama, T.; Hashimoto, T.; Kajiya, K.; Kumazawa, S. Affinity of polyphenols for lipid bilayers. Biofactors 2000, 13, 147-151.

[14] Lo, Y.L.; Tsai, J.C.; Kuo, J.H. Liposomes and disaccharides as carriers in spray-dried powder formulations of superoxide dismutase. Journal of Controlled Release 2004, 94 (2-3), 259-272.

[15] Chu, C.; Tong, S.; Xu, Y.; Wang, L.; Fu, M.; Ge, Y.; Y, J.; Xu, X. Proliposomes for oral delivery of dehydrosilymarin: preparation and evaluation in vitro and in vivo. Acta Pharmacologica Sinica 2011, 32 (7), 973-980. 